\title{
Asertividad en estudiantes participantes de la 'Gran Cruzada Andina' en favor de los damnificados del norte del Perú, 2017
}

\author{
Gareth Del Castillo Estrada ${ }^{1} \&$ Yanet Castro Vargas ${ }^{2}$ \\ 1.- Magíster en Psicología Organizacional \\ Máster en Business Administration \\ Máster en Marketing Intelligence \\ 2.- Docente Principal del Departamento Académico \\ Magíster en Psicología. \\ Doctora en Ciencias de la Salud
}

\section{Resumen}

Cusco - Perú

Al considerar la importancia de establecer perfiles que permitan identificar el desarrollo de conductas adaptativas y socialmente valoradas en adolescentes, se hace necesario el compromiso de distintas instituciones vinculadas a esta población, entre las cuales la universidad se constituye en un importante escenario para el análisis teniendo en cuenta su constitución poblacional y, más aún, su naturaleza vinculada a la generación del conocimiento. La ocurrencia del fenómeno de El Niño costero, generó una serie de iniciativas vinculadas al apoyo a los damnificados, siendo la Universidad Andina del Cusco activa participante, con la organización de la 'Gran Cruzada Andina' en favor de los damnificados del norte del Perú, en el 2017. Situación que permitió la evaluación de conductas asertivas entre los participantes de esta actividad. Nos valimos de la entrevista como técnica para el desarrollo de la investigación, y de la encuesta, como instrumento de recolección. La investigación se desarrolló bajo un alcance descriptivo siendo su diseño no experimental de corte transversal; al realizarse solo durante el día la actividad ejecutada incrementándose el nivel de confianza del estudio, con una muestra mayor a los 600 estudiantes. Los resultados encontrados muestran una mayor agrupación de los evaluados en las categorías diagnósticas medias o promedio de la asertividad, encontrándose más del $50 \%$ de la población evaluada en dicha categoría, además, se refleja la tendencia de la muestra evaluada, a ocupar niveles inferiores en contraste a superiores de asertividad; respecto a la evaluación de extremos. Por otro lado, en la comparación de diferencias en la presentación de la variable por género, no se encuentra diferencia estadística significativa, presentándose la asertividad de igual manera en varones y en mujeres dentro de la muestra evaluada. Se concluye que los niveles altos de asertividad no son representativos de la población en evaluación, se encontró coherencia con estudios que plantean que esta variable — no necesariamente - se presenta relacionada con el apoyo u orientación social, sino más bien con condiciones que pueden y son inherentes a estados individuales.

Palabras clave: asertividad; habilidades sociales; estudiantes. 


\section{Abstract \\ Assertiveness in students participating in the Great Andean Crusade in favor of the victims of northern Peru, 2017}

The present research aimed to consider the importance of establishing profiles to identify the development of adaptive and socially valued behaviors in adolescents. It is necessary the commitment of different institutions related to this population, among which the university constitutes an important scenario for the analysis, taking into account its population constitution and even more its nature linked to the generation of knowledge. The occurrence of the so-called coastal El Niño phenomenon, generated a series of initiatives linked to support for victims, with the Universidad Andina of Cusco active participant, with the organization of the Great Andina Crusade in favor of the victims of northern Peru in 2017. A situation that allowed the evaluation of assertive behaviors among the participants of this activity. Using the interview as a technique for the development of the research and of the survey as a collection tool, the research was developed under a descriptive scope, its nonexperimental design being cross-sectional when the activity was performed only during the day. The results show a greater grouping of the evaluated in the average or average diagnostic categories of the assertiveness, being found more than $50 \%$ of the population evaluated in that category, in addition it is reflected the tendency of the sample evaluated to occupy inferior levels in contrast to Superior assertiveness, regarding the evaluation of extremes. On the other hand, in the comparison of differences in the presentation of the variable by gender, no statistically significant difference was found, presenting the same assertiveness in males as in females, within the sample evaluated. In conclusion, high levels of assertiveness are not representative of the population under evaluation, being consistent with studies that suggest that this variable is not necessarily related to social support or orientation, but rather to conditions that may and are inherent to individual states.

Key words: assertiveness; social skills; students. 


\section{Introducción}

La asertividad se presenta como una variable de amplio interés para investigadores, situación que podría deberse a su clara vinculación con otras variables que permiten un mejor entendimiento de la conducta social adaptativa o por el reciente interés en establecer conductas asertivas en poblaciones vulnerables y trabajar sobre las mismas para disminuir los riesgos. El estudio de Gaeta \& Galvanovskis, del 2009 muestra, tras desarrollar una serie de análisis de orden factorial a distintos instrumentos de evaluación de las habilidades sociales que no presentan diferencia respecto a otros instrumentos que miden la variable 'asertividad', de esta manera, se comprueba que existen problemas al distinguir las habilidades sociales de las habilidades asertivas (Aguilar, 1995), esto teniendo en cuenta que las conductas asertivas contemplan distintos elementos evidenciables en las conductas propias de la habilidad social. Muestra de esto es el adecuado uso de los procesos de comunicación según ámbitos y agentes de los participantes. Es, además, importante considerar que otros autores relacionan la asertividad con multiplicidad de variables, siendo una de ellas la autoestima, dentro de estos (Rodriguez \& Serralde, 1991), plantean que hablar de asertividad es lo mismo que hablar de autoestima o, por lo menos, se constituye en un elemento de la misma. En este entender sería prudente considerar la multidimensionalidad de dicha variable, así como de aquellas con las cuales es sujeta a comparación, puesto que es posible acomodar las relaciones no directamente entre variables globales, sino entre factores componentes de las mismas. Dentro de las actuales consideraciones sujetas a mención está la vinculación de la asertividad y la agresividad, relación que se plantea desde la presentación del modelo (Wolpe, 1977), al considerar la asertividad como una conducta más o menos agresiva que investigaciones posteriores tratarán de identificar, sin embargo, la intensidad, frecuencia y otros criterios de medición permitirán establecer una relación más clara entre dichas variables.

Si bien se debe tener en cuenta la dificultad de definir la asertividad, para el presente estudio se entiende —en razón a la manifestación de comportamiento que permite enfrentarse con éxito a situaciones difíciles_-, de igual manera que a sí mismo y a los demás; en este entender veremos que una conducta asertiva muestra la integridad del individuo, al revelar preocupación por los otros y generar, paralelamente, mejora en las relaciones interpersonales (Universidad Politécnica de Valencia, 2014). Se asume dicha postura tomando en cuenta las condiciones para el desarrollo del presente estudio siendo un escenario particular de análisis, en el cual se desarrolla la recolección de datos. En este entender veremos que aquellas consideraciones que vinculan la conducta asertiva con la posibilidad de enfrentar situaciones difíciles y la preocupación por otros acomodan, claramente con el estudio, siendo una adecuada guía para su ejecución.

Teniendo en cuenta el fenómeno de El Niño costero y su incidencia en el Perú, fenómeno que se caracteriza por el calentamiento anómalo del mar adyacente a la costa del país, que 
origina una serie de desequilibrios naturales que dieron como consecuencia la declaración del estado de emergencia en distintas regiones del Perú, tal es el caso de Tumbes, Piura o Lambayeque, dicho escenario originó perjuicio, no solo en una serie de ámbitos — tal es el caso de: deterioro en infraestructura, pérdida de actividad laboral, migración de localidades y otras pérdidas de orden material—, sino, muy a pesar de todo el país, fallecieron más de un centenar de personas, más de 100000 damnificados, además, muchos desaparecidos y heridos. En este panorama se establecieron una serie de actividades vinculadas a prestar apoyo a la población del norte del país, la más afectada por causa de este fenómeno, estando comprometidas con dicha causa, distintas instituciones y empresa del país y el extranjero, siendo la Universidad Andina del Cusco una de ellas, que mediante la 'Gran Cruzada Andina' en favor de los damnificados del norte del Perú, se recabaron víveres y demás insumos.

Los espacios de apoyo y colaboración con poblaciones afectadas y otras de riesgo, se constituyen en ámbitos idóneos para el desarrollo de distintas actividades, siendo el objetivo de la investigación el determinar si la asertividad se constituye en una de las características o integra el perfil de los participantes y, de esta manera, conocer un poco más de aquellas particularidades que permitirían, a la larga, promover un desarrollo sostenido de conductas de apoyo y responsabilidad social en estudiantes, por otro lado, variables como la asertividad se constituyen en interesantes al generar una serie de relaciones con otras variables de estudio, las mismas que abordan temas de contacto social o habilidades relacionales.

\section{Materiales y métodos}

El estudio se inició al evaluarse la magnitud de los eventos del fenómeno de El Niño costero en el Perú y, comprobando el interés de la Universidad Andina del Cusco por programar una actividad que permitiera el apoyo de damnificados del norte del país, Se iniciaron los trámites vinculados a las autorizaciones pertinentes a nivel de la Escuela Profesional de Psicología de la Universidad, para el uso de recursos materiales a nivel de instrumentos de evaluación y participación de alumnos que se encontraran desarrollando asignaturas vinculadas a la investigación, dirigidas hacia su respectiva capacitación vinculada al manejo de instrumentos y vaciado de datos.

El estudio se desarrolla bajo un enfoque cuantitativo el cual se fundamenta en la medición que se lleva a cabo al utilizar procedimientos estandarizados y aceptados por una comunidad científica, su alcance es descriptivo, puesto que busca especificar propiedades y características importantes referidas a la asertividad en la población de estudio, siendo su diseño no experimental-transversal, al no manipular de manera deliberada alguna variable, observándose la asertividad en un ambiente natural para después analizarla; transversal porque se recolectan datos en un tiempo único. Su propósito es describir las variables y analizar su incidencia e interrelación en un momento dado. (Hernández, Fernández, \& Baptista, 2014). 
La recolección de información se inició con el análisis del modelo teórico que permitiría interpretar la naturaleza y operacionalización de la variable objeto de estudio, encontrándose la teoría de Rhatus, la misma que sirve de modelo para el desarrollo de la «Escala de asertividad de Rathus (RAS)», que según Díaz, Ruiz \& Villalobos (2012), se presenta como uno de los instrumentos ampliamente utilizados en la evaluación y diagnóstico de la conducta asertiva.

Una vez seleccionado el instrumento e identificada la entrevista como técnica de recolección de datos se procedió a evaluar a los estudiantes durante el desarrollo de la denominada «Gran Cruzada Andina», actividad que convocó a estudiantes de las cinco facultades de la universidad, organizados por las escuelas profesionales en la plaza Túpac Amaru, del distrito de Wánchaq. Para la determinación del tamaño de la muestra, se utilizó un nivel de confianza del $99 \%$, siendo lo usual un nivel del $95 \%$, habiéndose convenido en dicho nivel para reducir la incertidumbre en el estudio, y teniendo en cuenta la posibilidad de evaluar sin limitaciones de orden operativo a los estudiantes en la actividad de apoyo.

\section{Resultados}

Los resultados presentados en esta sección buscan responder los objetivos planteados para el estudio, encontrándose entre los mismos la identificación y descripción de los niveles de asertividad en la muestra de estudio y la determinación de diferencias en la presentación de dicha variable, según género.

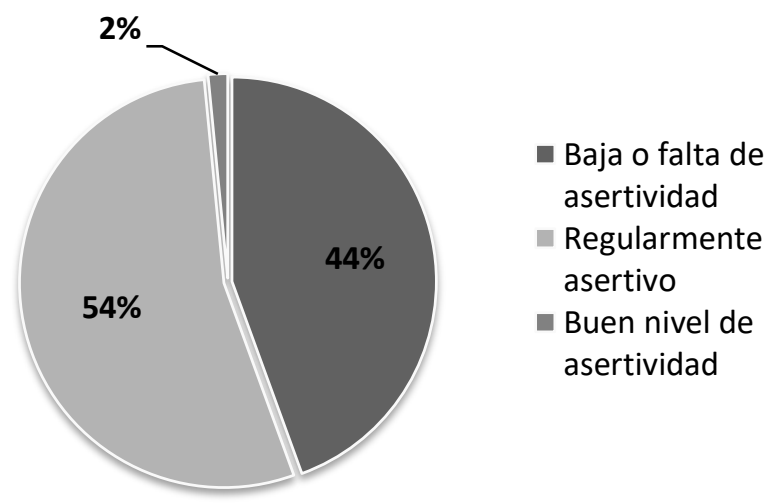

Figura 1. Asertividad en estudiantes participantes de la «Gran Cruzada Andina» en favor de los damnificados del norte del Perú, 2017.

Conforme los resultados del análisis de datos presentados en la figura 1, se puede apreciar que los estudiantes participantes de la «Gran Cruzada Andina» en favor de los damnificados 
del norte del Perú, desarrollada en el 2017, se concentra mayormente en la categoría 'regularmente asertivo', con un 54 \% (342 estudiantes), mientras la categoría 'baja o falta de asertividad' se constituye en aquella con segunda categoría que recoge un $44 \%$ (282 estudiantes), siendo la categoría 'buen nivel de asertividad' aquella que agrupa a un menor número de encuestados, con solo el $2 \%$ (10 estudiantes). De esta manera se podría considerar que los estudiantes que participaron de la «Gran Cruzada Andina», presentan puntajes que los sitúan, por mayoría, en la categoría media o promedio, no siendo la asertividad una variable que constituya una característica relevante en ellos.

Como procedimiento previo a la comparación entre grupos de investigación, es decir, entre alumnos varones y mujeres de la Universidad Andina del Cusco, se procedió al análisis de la bondad de la curva normal a través de la prueba ' $Z$ de Kolmogorov Smirnov', esto permitió determinar la conveniencia de utilizar una prueba no paramétrica para el estudio, al obtenerse un $P$ Valor de $0,00<0,05$ que difiere significativamente de la distribución normal; teniendo en cuenta lo mencionado, así como el carácter ordinal de los datos en análisis, se decidió realizar el análisis comparativo utilizando una estadística no paramétrica, es el caso de la ' $U$ de Mann-Whitney', prueba alternativa a la 'T de student.'

Tabla 1. Prueba de normalidad Z de Kolmogorov Smirnov, para resultados de asertividad

Z de Kolmogorov Smirnov

,056
$\boldsymbol{P}$

, 000

En el $95 \%$ de confiabilidad, al utilizar la prueba estadística 'U de Mann-Whitney', podemos afirmar que la asertividad entre estudiantes varones y mujeres de la Universidad Andina, que participaron en la «Gran Cruzada Andina», no presenta diferencia estadística significativa para el periodo contemplado en el estudio, es decir 2017, teniendo en cuenta una significación asintótica bilateral o $\mathrm{P}$ Valor de $\mathrm{p}=0,51>0,05$, de esta manera, se rechaza la hipótesis de investigación y se acepta la hipótesis nula. 
Tabla 2. Niveles de asertividad según género en estudiantes que participaron de la «Gran Cruzada Andina» en favor de los damnificados del norte del Perú, en el 2017.

\begin{tabular}{|c|c|c|c|c|c|c|}
\hline \multirow[t]{3}{*}{ Asertividad } & \multicolumn{4}{|c|}{$\begin{array}{c}\text { Estudiantes de la Universidad } \\
\text { Andina del Cusco } \\
\end{array}$} & \multirow{2}{*}{\multicolumn{2}{|c|}{ Total }} \\
\hline & \multicolumn{2}{|c|}{ Varones } & \multicolumn{2}{|c|}{ Mujeres } & & \\
\hline & $f$ & $\%$ & $f$ & $\%$ & $f$ & $\%$ \\
\hline Alta & 1 & - & 9 & 3 & 10 & 2 \\
\hline Regular & 163 & 57 & 179 & 52 & 342 & 54 \\
\hline Baja & 124 & 43 & 158 & 46 & 282 & 44 \\
\hline Total & 288 & 100 & 346 & 100 & 634 & 100 \\
\hline $\begin{array}{l}\text { U de Man-Whitney = } \\
36301.0\end{array}$ & & & & & & \\
\hline
\end{tabular}

\section{Discusión}

En el escenario de desarrollo del presente estudio, se consideró importante llegar a conocer más a profundidad sobre aquellas características que presentaban los estudiantes participantes de la «Gran Cruzada Andina», en este ánimo se escogió la variable 'asertividad' para su identificación y descripción en la muestra de estudio, teniendo en cuenta su naturaleza y relación con otras variables vinculadas al comportamiento social. Tras el correspondiente análisis de los datos propios del estudio se pudo encontrar, que el desarrollo de niveles altos de asertividad no se presentan como característicos del perfil del estudiante andino durante la «Gran Cruzada Andina», en este entender - y respecto a la definición de asertividad dada por la Universidad Politécnica de Valencia, en el 2014-, veremos que la asertividad se representaría mejor al analizarla como tendiente a resguardar la integridad del individuo más que como preocupación por otros.

Por otro lado, puesto que se establece en distintos estudios como el de Londoño \& Valencia, de 2008, es más característica la vinculación de la asertividad con conductas que permitan la manifestación de opiniones individuales o la resistencia a la presión de grupo, que con aquellas asociadas al comportamiento responsable a nivel social.

Por último, en relación a la presentación de la conducta asertiva por género, al no encontrarse diferencia estadistica significativa se comprueba lo encontrado por Ramírez, Álvarez, Cadena, Mendoza, Alarcón, \& García, en el 2016, en su estudio de asertividad frente al consumo de alcohol y tabaco, respecto a la existencia de conductas asertivas de igual intencidad y frecuencia en adolescentes de ambos sexos. 


\section{Conclusiones}

Los niveles altos de asertividad no son representativos de la población evaluada, encontrándose coherencia con estudios que plantean que esta variable, no necesariamente, se presenta relacionada con el apoyo u orientación social, sino más bien, con condiciones inherentes a estados individuales.

No se considera necesaria la presencia de niveles desarrollados de asertividad en la presentación de conductas de apoyo social, como la desarrollada por la Universidad Andina del Cusco, en apoyo a los damnificados del norte del Perú.

No existen claras diferencias en la presentación de la variable asertividad al analizarse por género, lo que nos llevaría a pensar que los recursos de comportamiento vinculados a la variable se presentarían de manera parecida en varones y mujeres, situación que podría relacionarse con ambientes respetuosos de la equidad de género en el ámbito académico estudiado.

\section{Agradecimientos}

A la psicóloga Liliana Peña Farfán, que en su calidad de docente del curso de Taller de investigación 2, de la Escuela Profesional de Psicología, colaboró con la recolección y codificación de datos para el desarrollo del estudio.

\section{Fuentes de financiamiento}

El estudio se desarrolló sin fuente de financiamiento externa, siendo los costos del proceso asumidos por los investigadores.

\section{Conflictos de interés}

Los autores declaran no tener conflictos de interés en la publicación de este artículo. 


\section{Referencias Bibliográficas}

Aguilar, E. (1995). Estandarización de la escala de asertividad de Michelson y Wood en una muestra mexicana de niños de 8 a 16 años. Tesis inédita de Licenciatura en Psicología: Universidad Nacional Autónoma de México.

Bryant, B., \& Trower, P. (1974). Social difficulty in a student sample. British Journal of Educational Psychology, 13-21.

Hernández, R., Fernández, C., \& Baptista, P. (2010). Metodología de la investigación (Quinta ed.). Mexico D.F.: Mc Graw Hill.

Hernández, R., Fernandez, C., \& Baptista, P. (2014). Metodología de la investigación. México D.F.: Mac Graw Hill.

Gaeta, L., \& Galvanovskis, A. (2009). Asertividad un análisis teórico - empírico. Enseñanza e investigación en psicología, julio-diciembre, 403-425.

Londoño, C., \& Valencia, C. (2008). Asertividad, resistencia a la presión de grupo y consumo de sustancias en universitarios. Acta colombiana de psicología , 155-162.

Riso, W. (1987). La percepción social en la conducta asertiva. Revista Análisis del Comportamiento, 285-295.

Rodriguez, E., \& Serralde, M. (1991). Asertividad para negociar. México D.F.: McGraw-Hill Interamericana de México, S.A de CV.

Universidad Politécnica de Valencia. (2014). Asertividad. Valencia: Universidad Politécnica de Valencia. 